\title{
UNIVERSIDAD E INVESTIGACIÓN EN EL CONTEXTO DEL MERCADO DEL CONOCIMIENTO
}

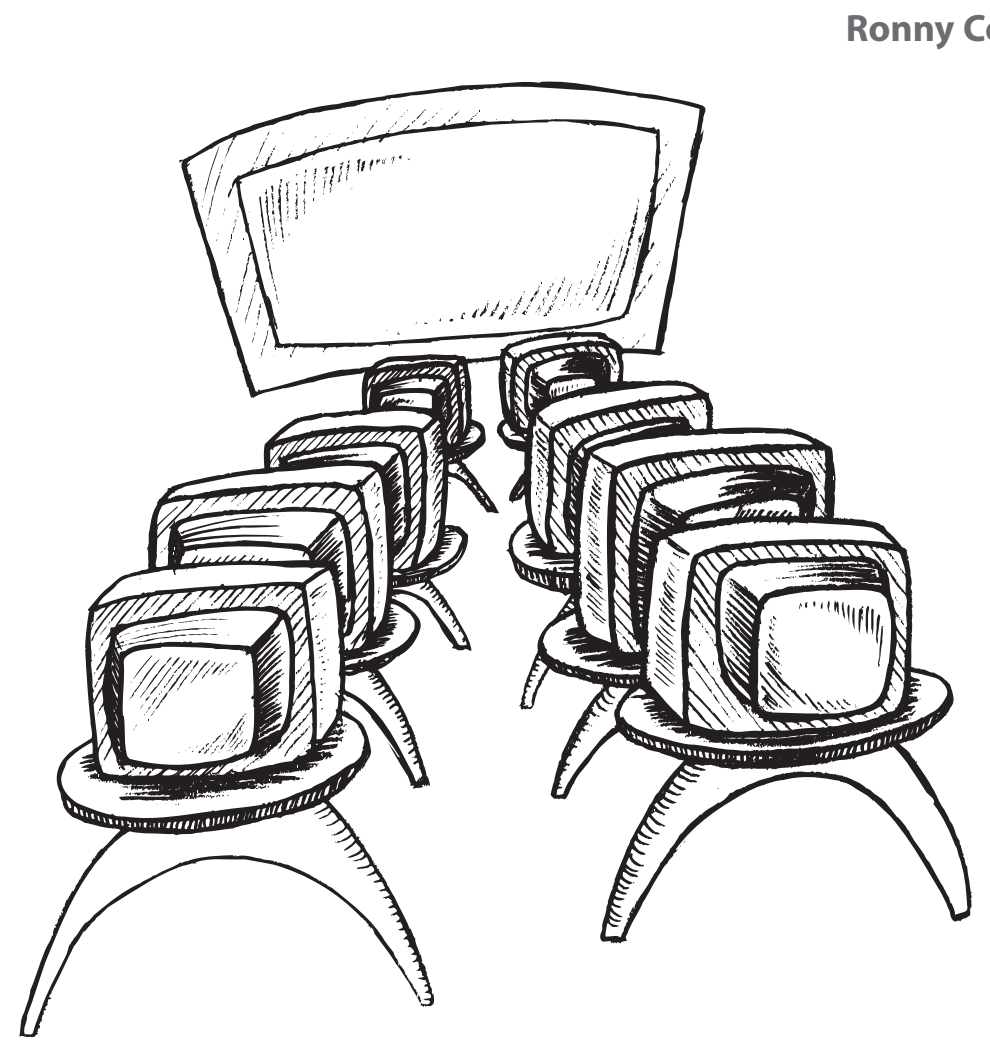

\section{Introducción}

Las tecnologías de la información y comunicación y su notable influencia sobre la ciencia y tecnología -sobre todo en electrónica, biotecnología y materiales- han ocasionado profundos cambios, no sólo en los sistemas productivos e industriales, sino en la estructura económica y social de todos los países; el sector de la educación y las Universidades obviamente, no escapan a esta realidad, las actividades de estas últimas y sus la-

* Director General de Investigación y Transferencia de Tecnología de la Universidad Técnica Particular de Loja- Ecuador. 
bores de investigación se desarrollan en la llamada sociedad de la información en la cual acabamos de entrar, y difieren sustancialmente de la sociedad industrial que la ha precedido. En la sociedad industrial el principal recurso era la energía y sus herramientas eran artefactos como carretillas, elevadores, grúas, trenes, automóviles y aviones. Su primordial característica fue que nos permitió expandir el cuerpo humano. En la actualidad, la sociedad de la información difiere en que la velocidad de su evolución es mucho mayor y su recurso mayor es la información. Ésta constituye un recurso muy especial, ya se ha señalado, en más de una ocasión, que puede ser ingrávida, invisible y estar al mismo tiempo en varios lugares. Las herramientas de la sociedad de la información permiten crear, almacenar, enviar, manipular y transformarla. Pero sobre todo, la principal característica de la revolución de la información es que nos va permitir expandir nuestro intelecto (Jones, 1996).

Es a finales de los noventa en donde conocimiento e información comienzan a perfilarse como compo- nentes principales que delinean un nuevo sistema en el mundo entero, las economías no están basadas únicamente en la acumulación de capital físico y recursos humanos; es necesario también un sólido cimiento de información y aprendizaje, lo que distingue a los pobres -sean personas o países- de los ricos es no sólo que tienen menos capital, sino también menor conocimiento (Banco Mundial, 1999). En el año 2005 dicha aseveración es confirmada al intentar explicar las diferencias de productividad y competitividad entre los países, el factor principal no es la abundancia de recursos naturales ni el capital; es el conocimiento, específicamente, y de manera concreta traducido en tecnologías nuevas e innovadoras que facilitan producir más con menos. En los países desarrollados, la creación y adopción de nuevas tecnologías explican cerca de la mitad de todo el crecimiento económico (Banco Mundial, 2005).

Es en esta transición hacia la sociedad del conocimiento ${ }^{1}$ donde se originan particulares sistemas socioeconómicos de relaciones, interdependencia e intercambio, ca-

1 Silvio, José. en “La virtualización de la Universidad" (2000) IESALC-UNESCO manifiesta que “... estamos aún en la etapa de la sociedad de la información, como antesala a la más sofisticada sociedad del conocimiento. En la actualidad, coexisten diversas configuraciones de la sociedad, unas más aproximadas que otras a una sociedad del conocimiento, como en todo período de transición". 
da uno con características singulares y funciones, cuya especificidad es delimitada por el rol que cumplen los distintos actores y sistemas. Uno de los sistemas generados en este contexto y en el cual las Universidades y la investigación son elementos primordiales es el mercado del conocimiento.

Desde el punto de vista socioeconómico simple, el término "mercado" nos invita a pensar de forma general en un entorno en el que participan oferentes y demandantes los que realizan acuerdos de intercambio de bienes y/o servicios y de flujos monetarios, este espacio de intercambio puede o no ser de orden internacional dependiendo de la tipología de los bienes sujetos de transacción y de las necesidades de demanda o de producción de los distintas regiones o países. Cuando de este mercado descrito anteriormente se configura uno nuevo del que forman parte las Universidades, las Tecnologías de la Información y Comunicación (TIC's), innovación y conocimiento, la simplicidad del análisis anterior queda minimizado en su campo de explicación y acción, debido a la complejidad que estos nuevos agentes, en lo que concierne a su función, dinámica y relaciones complejas que engrana el nuevo mercado del conocimiento.

\section{La principal característica de la revolución de la información es que nos va a permitir expandir nuestro intelecto.}

El nuevo mercado del conocimiento tiene características especiales, muchos estudiosos y organismos del tema censuran de diversas maneras las formas y las asimetrías en las que las Universidades -en especial latinoamericanas- participan en este sistema, las críticas son varias: desde la participación de Universidades corporativas internacionales en la oferta de programas educativos a nivel de pregrado y posgrado, lo que han llamado la "MacDonalización" de la educación superior, la brecha existente en el uso de tecnología y los recursos presentes entre los consorcios universitarios del Norte y del Sur; el tema de la educación superior en las rondas de negociación del Comercio Mundial; incluso en poner en tela de juicio si los programas ofrecidos a Distancia y por Campus Virtual tienen la calidad de los ofrecidos por las Universidades presenciales. 
Pero la realidad es que las Universidades latinoamericanas están desde hace mucho tiempo involucradas en este mercado del conocimiento, Kart Larsen, Director del Centro de Investigación e Innovación Educativa de la OCDE señala que "El mercado de la enseñanza superior también ha estado presente por mucho tiempo, si bien no se lo conocía por ese nombre" (UNESCO, 2002). Pero ¿cuál es el elemento esencial para la configuración de la sociedad de la información y del mercado del conocimiento?, desde mi punto de vista es la INNOVACIÓN -o la ausencia de esta-, ya que por ello las Universidades y los consorcios empresariales-Universidades han decidido ofertar nuevas opciones en el ámbito educativo, de igual manera las TIC's y su aplicación son una forma de innovación, los demandantes particulares, gubernamentales y empresariales también buscan niveles de formación y capacitación que les permita innovar en sus labores cotidianas con el afán de ser productivos y competitivos.

Joseph Schumpeter, uno de los más notables economistas de todos los tiempos, considera que la innovación es el factor básico de desarrollo económico. Obviamente, la concepción de Schumpeter acerca de la innovación y de su indispensable presencia no se limita a inventos patentados o a condiciones de merca- do, toma en consideración nuevas combinaciones de organizaciones: en los métodos de gestión, construcción de nuevas estructuras organizativas; nuevas alianzas y sinergias entre industria, estado y escuela -llámese ahora Universidades-. Todos estos aspectos citados anteriormente son transversales a las funciones propias de la Universidad y su rol de generador, catalizador, de ciencia y desarrollo en la sociedad (Correa, 2006). Las deficiencias de la Universidad en servir a la sociedad, su falta de "pertinencia", el impacto en las instituciones de educación superior y en las Universidades de las políticas del Banco Mundial y de la visión con ánimo de lucro, son algunas de las razones que llevan a muchos -desde distintas posiciones ideológicas y latitudes- a plantear su actual crisis. Sin embargo, en la última década se ha venido promoviendo, en especial por la UNESCO, de forma multilateral y también por los gobiernos de diversos países de manera unilateral, el debate, reflexión y adopción de acciones, que tienen como objetivo convertir la denominada "crisis" de la Universidad, en una oportunidad para reinventar las instituciones de educación superior y adecuarlas a las exigencias de la sociedad del conocimiento. Este proceso ha dado lugar a la transforma- 
ción de instituciones tradicionales en Universidades innovadoras (López, 2006).

Es en este contexto, el objetivo de este ensayo es reflexionar y participar a la comunidad universitaria de la aplicación y resultados de un modelo de gestión innovador puesto en marcha en la Universidad Técnica Particular de Loja (UTPL), desde sus dos principales dimensiones los Centros de Investigación Transferencia de Tecnología Extensión y Servicios (CITTES) y la Modalidad de Estudios a Distancia, en las que el 'Espíritu' y los 'Valores' son el eje transversal del accionar universitario.

\section{Los centros de investigación, trasferencia de tecnología extensión y servicios (CITTES)}

Iniciaré este acápite recordando un comentario realizado por el doctor Juan de Dios Vial Correa, ex Rector de la Pontificia Universidad Católica de Chile, que manifestó lo siguiente: "Si pudiéramos viajar en una máquina del tiempo y recorrer Latinoamérica hacia épocas anteriores, y ubicarnos en el siglo XIX, de seguro nos causaría asombro las cos- tumbres y las relaciones e interacciones presentes en esa época de la humanidad. Sin embargo, si programamos la máquina del tiempo para que nos ubique a mediados del siglo XIX, en una de las Universidades de ese entonces, la verdad es que no sentiríamos mayor diferencia en los procesos de gobierno, gestión y proceso de enseñanza aprendizaje de las Universidades, es más, en poco tiempo nos sentiríamos como en casa"2.

A pesar de que en muchas Universidades latinoamericanas se ha emprendido en procesos de reforma, no es menos cierto que esas buenas intenciones han sido opacadas y distorsionadas por las "modas" imperantes en distintos períodos de la sociedad, entre ellas tenemos: reformas y contrarreformas curriculares, cambios de pensum, metodologías de estudio, planeación estratégica, la calidad total, etc., todas ellas herramientas para la evolución y cambio de paradigma, sin embargo, dentro del academicismo y ostracismo imperante a lo interno de la Universidad se convierten en fines mismo de la reforma, transformándose estas iniciativas en un círculo perverso, deformando así el concepto y los objetivos 
de innovación en las instituciones de educación superior; ciertamente en las Universidades latinoamericanas aún no está clara la definición y el concepto entre Novedad (que involucra un cambio superficial) y el de Innovación (que implica un proceso duradero, de alto índice de utilización y mejoras sustanciales), la Asociación Nacional de Universidades e Instituciones de Educación Superior (ANUIES-México) identifica cinco ámbitos de innovación: 1) planes y programas de estudio; 2) proceso educativo; 3 ) uso de la tecnologías de información y comunicación; 4) modalidades alternativas para el aprendizaje; 5) gobierno dirección y gestión; concordantes con esta apreciación los cambios y las estrategias a lo interno de la Universidad y con su entorno deben ser integrales y sistémicas para que se asimile y discierna los impactos de globalización y de las tecnologías de la información en la formación de conocimientos y de capital intelectual.

En la mayoría de las Universidades latinoamericanas el proceso educativo y su "resultado final" -hablando figuradamente en términos de mercadolos profesionales al servicio de la sociedad, dependen de los insumos utilizados en las distintas fases sucesivas de dicho proceso, es obvio suponer que si se utilizan materias de baja ca- lidad el resultado no será el mejor, en efecto. ¿Cómo esperamos que nuestros recién titulados tengan un nivel acorde a estándares internacionales, si uno de sus "insumos" sus profesorestutores adolecen en términos generales de una cultura de investigación, un ciego que guiando a otro ciego, están condenados al hoyo (Mateo, 15), y su entorno universitario tampoco es el más idóneo?

Del total de IES (Instituciones de Educación Superior) existentes en la región -refiriéndose a América Latina- seguramente una fracción no superior al 3\% podrían denominarse "Universidades de investigación" (Research Universities). Junto a ellas existe otro grupo (tal vez un $7 \%$ ) de Universidades con investigación "que en realidad no alcanzan a situarse en el nivel de internacionalización que supone participación en la comunidad mundial de producción científica y tecnológica. En cambio, las demás instituciones -más de un 90\%- son puramente docentes" (Brunner, 2003).

Los principales indicadores de Ciencia y Tecnología (C\&T) a nivel latinoamericano permiten describir cuantitativa y cualitativamente el desarrollo de la ciencia y el rol de sus actores en nuestros países; es así que el promedio de inversión latinoamericano en C\&T, como porcentaje del PIB es igual al 0,62\%; en el Ecuador, este 
indicador llega al 0,07\% del PIB, similar al de Nicaragua, y muy por debajo del de Bolivia con el 0,26\%. En el país en el año 2003 hubo cerca de 845 profesionales trabajando en investigación científica y tecnológica, pero sólo un $10 \%$ estába dedicado a tiempo completo a dichas actividades y, un $43 \%$ investigaba menos de la mitad de su jornada laboral (FUNDACYT, 2003). Correlacionando hipotéticamente este dato a la Universidad ecuatoriana podemos extrapolar y concluir que: menos de 90 personas que realizan investigación a tiempo completo -no necesariamente doctores- están en las Universidades.

Otra de las limitaciones importantes para el desarrollo de la ciencia en las Universidades ecuatorianas es la titulación de tercer y cuarto nivel de los docentes, así como su dedicación exclusiva para la docencia e investigación. Según datos del año 2000, de los cerca de 17.000 docentes que imparten clase en las Universidades, menos del $20 \%$ de ellos, es decir cerca de 3.000 , poseen titulación de tercer nivel, y apenas el $1 \%$ titulación a nivel de doctorado. Igualmente, solamente el $12 \%$ del total de docentes de las Universidades se dedican a tres cuartos de tiempo y tiempo completo a actividades inherentes al quehacer universitario. Para el año 2004, el panorama no cambia, “...un estudio del Consejo Nacional de Educación Superior, advierte que en Ecuador, alrededor de 172 ecuatorianos han obtenido el grado académico de PhD (CONESUP, 2004), el estudio sin embargo no menciona si estos 172 doctores se encuentran desarrollando actividades de docencia y/o investigación en las Universidades o en centros de investigación afines.

Con estos datos, no es extraño entonces, estimar en porcentaje que en Latinoamérica y en Ecuador -salvo honrosas excepciones-, de las actividades desarrolladas dentro de las Universidades, tanto profesores como estudiantes dedican más del 95\% de su tiempo a la docencia y menos de un $5 \%$ a la investigación y extensión (Romero, 2006). Esta realidad traducida a indicadores de C\&T permite ratificar la valoración, de los Indicadores de Ciencia y Tecnología proporcionados por RICYT (http://www.ricyt.org) para el año 2004, de los artículos científicos por cada cien mil de habitantes registrados en SCI SEARCH, uno es de Ecuador, 19 de Chile y 124 de Estados Unidos.

Algunas de las tendencias hacia las que parece moverse la investigación universitaria son las siguientes: generación de nuevos conocimientos altamente valorados por la sociedad; internacionalización creciente 
Ronny Correa Q.

de la investigación que cada vez más requerirá de la cooperación transnacional; dependencia creciente de fuentes de financiamiento externas a la Universidad; la innovación como factor principal de la competitivi$\mathrm{dad}$, tendiendo a favorecer la interacción entre investigación universitaria, sector productivo y entorno tecnológico; la formación de recursos humanos implicará un mayor esfuerzo en la formación de jóvenes investigadores y su mayor movilidad; tensión entre las necesidades inmediatas que enfatizan la investigación aplicada -dada la necesidad de obtener financiamiento- y las de más largo plazo que defienden la investigación básica; desarrollo de gabinetes de predicción de tendencias y necesidades tecnológicas claves para el desarrollo de la sociedad; y fusión de la cultura científica y de la

En poco más de un año hemos pasado a tener más de 160 de nuestros jóvenes profesores como estudiantes de doctorado en diversas Universidades de Europa y América. humanística (López, 2006). Reseñamos como se aproxima a comentarios a la estrategia de la UTPL.

En la Universidad Técnica Particular de Loja, como también se está dando en otros contextos, hemos venido desarrollando una serie de incubadoras de investigación a las que denominamos Centros de Investigación, Transferencia de Tecnología, Extensión y Servicios (CITTES) http://www.utpl.edu.ec/cittes, que son una réplica adaptada a nuestro contexto de los Departamentos, Laboratorios, Institutos, etc., de las Universidades de los países desarrollados, sólo que con una estrategia que permite superar las difíciles fases iniciales. En estos CITTES, 432 jóvenes profesores, investigadores a tiempo completo, trabajan dos terceras partes de su tiempo en proyectos de investigación, muchos de ellos aplicados, dedicando un tercio de sus horas a la docencia en las diferentes Escuelas, de forma presencial o a distancia, ya que la modalidad a distancia es en nuestra Universidad transversal a las diferentes escuelas. Los CITTES van estableciendo relaciones con Departamentos, su similar de Universidades desarrolladas, con los que inician proyectos de investigación conjuntos. Nuestros jóvenes profesores realizan sus doctorados en esos Departamentos de 
Universidades de gran nivel, pero con la modalidad "sándwich" o de tiempo compartido, por lo que nunca dejan de estar en sus correspondientes CITTES por largos períodos de tiempo, que es el factor fundamental asociado a la fuga de cerebros. Naturalmente, la Educación a Distancia y un uso intenso y transparente de la tecnología puede ayudar mucho. En poco más de un año hemos pasado a tener más de 160 de nuestros jóvenes profesores como estudiantes de doctorado en diversas Universidades de Europa y América, con un presupuesto unitario del mismo orden de magnitud que todas las Universidades del país (Romero, 2006).

En la UTPL hemos cambiado no sólo nominativamente, sino en concepto el papel del clásico estudiante por el de profesional en formación; a partir de la mitad de la carrera, los profesionales en formación pasan la tarde o la mañana "trabajando" en los Centros de Investigación, Transferencia de Tecnología Extensión y Servicios (CITTES), a cargo de algún profesor docente-investigador, en algún proyecto real, obviamente de menor a mayor complejidad. De esta forma los profesionales en formación van adquiriendo las competencias prácticas de relación, responsabilidad, trabajo en equipo, etc., ne- cesarias para su desempeño profesional, y a la vez están preparándose con una experiencia directa para una futura actividad de investigación. Estos equipos mixtos con los estudiantes incrementan nuevamente el "Efecto Mateo" de nuestros grupos. En la actualidad, de los 3.000 estudiantes de modalidad presencial en la UTPL, el $45 \%$ de ellos realizan labores en los 23 CITTES, según su área de estudio.

\section{Modalidad a distancia y la unidad de capacitación continua}

El proveer una educación superior de calidad, pertinente a las necesidades de la sociedad, asequible, accesible y equitativa, a menor costo, con mayor cobertura geográfica, permanente e independiente en el tiempo y en el espacio, sin fronteras ni barreras, centrada en el estudiante, son los postulados y lineamientos -que a partir de finales de los noventa- se han definido y socializado en los distintos encuentros mundiales y regionales sobre educación superior y el papel de las Universidades en su relación con la humanidad. Sin embargo, ya en la década de los setenta es en donde la Educación a Distancia $(\mathrm{EaD})$ cumple con estos requerimientos y compromisos con la socie- 
dad; como se puede apreciar en el cuadro adjunto, esta "innovación" del sistema de educación superior tiene sus orígenes en la década de los cincuenta, y es en 1976, teniendo como pionera a la Universidad Técnica Particular de Loja (Ecuador), que esta modalidad está presente en Latinoamérica.

\section{Educación Superior a Distancia en el Mundo}

1. Open University of South Africa (1951)

2. British Open University (1969)

3. Universidad Nacional de Educación a Distancia, España (1972)

4. Everyman`s University, Israel (1974)

5. Allama Iqbal Open University, Paquistán (1974)

6. Universidad Técnica Particular de Loja, Ecuador (1976)

7. Universidad Estatal a Distancia, Costa Rica (1977)

8. Universidad Nacional Abierta, Venezuela (1977)

9. Anadolu University, Turquía (1981)

10. Open Universiteit, Holanda (1981)

11. Kyongi Open University, Corea (1982)

12. University of the Air, Japón (1983)

13. Universitas Terbuka, Indonesia (1984)

14. Indira Gandi National Open University, India (1985)

15. National Open University Taiwan. (1987)

16. Al Quds Open University Jordan, Jordania (1987)

17. Universidade Aberta, Portugal (1988)

18. Open Learning Institute of Hong Kong (1989)

* Elaborado a partir de Jones (2000)
La Modalidad Abierta y a Distancia de la UTPL, en la actualidad, hace presencia en todo el territorio nacional, con un modelo propio que sigue las líneas generales de los Sistemas de Educación a Distancia mundiales, pero con sus claves específicas que le permiten responder a las necesidades del país y de su gente, contribuyendo al desarrollo sociocultural de la comunidad. Desde el primer momento, la Modalidad Abierta y a Distancia ha sido coesencial al desempeño de la misión de la Universidad, por su función social, posibilitando el acceso a la educación superior a quienes por diversos motivos no pueden hacerlo de otra forma. Su desarrollo, en consonancia con las tendencias de nuestro tiempo, es tarea esencial en la Universidad y se acomete a través de sus órganos respectivos, teniendo en cuenta los diversos rasgos que la definen: separación física entre el profesor y los alumnos, la acción formativa basada en materiales impresos, audiovisuales, nuevas tecnologías y medios de comunicación, el aprendizaje flexible, independiente y autónomo, una comunicación bidireccional y multidireccional, la necesidad de apoyo tutorial y una red de Centros Universitarios para atender a una población dispersa geográfica- 
mente (García, 2002). Desde sus inicios en 1976, el número de estudiantes de la Modalidad a Distancia de la UTPL ha incrementado en un $1.200 \%$ (mil doscientos por ciento), actualmente cuenta con 21.540 estudiantes (20.710 en Ecuador y 830 en los centros internacionales), lo que demuestra con creces la gran acogida de esta propuesta universitaria. Otro dato interesante ha desatacar es el promedio de edad de las personas que estudian en la UTPLModalidad a Distancia, hoy por hoy el rango esta contemplado entre los 19 a 29 años de edad, hace aproximadamente cinco años atrás el promedio de edad estaba contemplado entre los 35 y 46 años de edad.

De la misma forma consideramos básico tener en cuenta los principios propios del sistema a distancia, ya que siguen vigentes, tanto o más que cuando nació esta modalidad, a pesar de que en estos momentos se acerquen a ella otros actores que no fueron privados de la educación superior en la etapa propia de sus estudios. Hoy en día, la incorporación de las nuevas tecnologías hace que la educación a distancia, además de atender a la necesidad de formación permanente, democratización de la educación o igualdad de oportunidades, pueda ser un componente valio- so, alternativa o complemento de la educación tradicional.

En estos últimos años, la incorporación de las TIC's, ha enriquecido enormemente a la $\mathrm{EaD}$ abriendo espacios nuevos para la interacción, la flexibilidad e inmediatez en la actualización de los contenidos y, en la asesoría a los alumnos. Esto debería, en principio, permitir superar las distancias de una forma enormemente efectiva; sin embargo, cuando se analiza en detalle la realidad de la llamada "modalidad virtual", al menos en el contexto latinoamericano, las realidades concretas suelen ser cientos de veces menores a la expectativa generada. Las razones son muy complejas, pero quizás la principal sea la falta de conexión de la oferta virtual con la realidad educativa como sistema integrado que va mejorando los procesos para facilitar el aprendizaje y la comunicación, y que toma como base los fundamentos teóricos básicos de la modalidad a distancia, de la cual la "modalidad virtual" no es más que una muy interesante variante. En cambio, se la ha visto como panacea que por sí sola solucionará los problemas o deficiencias que puedan darse en otros ámbitos del sistema educativo, o en su elemento principal, el modelo educativo (Rubio \& Romero, 2007). 
Ronny Correa Q.

\section{La Unidad de Capacitación Continua}

Las Universidades han cumplido hasta el presente una función de formación básica de científicos, tecnólogos y humanistas, pero su acción ha sido muy débil en la educación permanente de la población trabajadora profesional. Actualmente, las Universidades, especialmente las de los países en vías de desarrollo, están atravesando por una crítica situación motivada por factores de diversa índole, los que han debilitado su capacidad para asegurar esa formación básica. La educación permanente plantea a la educación superior una nueva exigencia de mayor magnitud que la formación básica, pues, para desempeñarse con éxito en el tipo de sociedad en la que se está desenvolviendo, necesitará cambiar sus concepciones y paradigmas de trabajo en materia de enseñanza y aprendizaje y otras actividades. Al mismo tiempo, deberán buscar nuevos socios y nuevas alianzas y trabajar de manera proactiva en un ambiente reticulado y pluralista, lo cual requerirá de cambios estructurales y funcionales profundos (Silvio, 2000).

Ya en 1969, Jorge Alberto Sábato definió el "triángulo de interacciones" o "triángulo de Sábato" en don- de el gobierno, sector productivo y las Universidades se interrelacionan creando alianzas estratégicas lo que da lugar a sinergias y estrategias de progreso de los países. Aunque las interacciones entre los tres actores tenían como objetivo el desarrollo tecnológico; la capacitación que podrían proveer las Universidades a las empresas y a los funcionarios del gobierno estaba implícita dentro de este modelo. En la actualidad, hablamos del "cuadrilátero competitivo" en donde el Estado, empresa, sectores sociales y Universidad, a través de acuerdos estratégicos se relacionan y vinculan la innovación, el aprendizaje, recurso humano e inclusión, de todos estos factores en la sociedad de la información. Una sociedad basada en conocimientos se puede desarrollar solamente por medio de relaciones cooperativas entre gobiernos, instituciones educativas, industria privada, instituciones financieras internacionales y organizaciones no gubernamentales (Banco Mundial, 2005).

En la UTPL, una de las formas de cooperación con el sectores sociales, empresas públicas y/o privadas es la que realiza nuestra Unidad de Capacitación Continua (http://www.utpl.edu.ec/capacitacion), la cual en estos últimos tres años ha capacitado a 21.700 personas en distintas áreas 
del conocimiento, las cuales han participado de programas y eventos de actualización y profesionalización. En la UTPL concebimos a la formación del recurso humano en las organizaciones, como un proceso integral permanente, acorde con los nuevos desafíos que imponen el entorno internacional, la globalización y el desarrollo del conocimiento que exigen nuevas necesidades de formación y actualización flexibles; en el cual las innovaciones en medios tecnológicos, metodologías y contenidos son elementos fundamentales de aporte desde nuestra Universidad hacia la sociedad.

\section{La ética y los valores}

"Los verdaderos problemas de la Universidad latinoamericana son una cuestión de ética y valores"3. Temas como ética, espíritu y valores, entendidos en su sentido más amplio, constituyen el núcleo de la temática universitaria del siglo XXI, especialmente en nuestro continente Latinoamericano..., centrarnos en los temas clave como los valores, la investigación y el servicio a la sociedad, usar de forma adecuada y transparente las TICs, y recrear densos contextos de interacción, colaboración y consenso. Las ciencias experimentales podrían estar cientos de veces más desarrolladas, si se fundamentaran en los aspectos profundamente humanos de las ciencias "experienciales", relacionadas con el tema de la unidad, dirección y sentido del ser humano y sus realizaciones sociales, culturales, económicas, políticas, espirituales... A fin de cuentas, las Universidades deberían ser, como crisol de las más grandes empresas humanas, "ciudades del amor", plenas de diálogo y colaboración, creadoras de sentido y humanismo, centros en los que la calidad sea ir, rompiendo nuestros propios límites, hacia los mayores horizontes en todas las dimensiones humanas (Romero, 2006).

3 Comentario emitido por el doctor Claudio Rama, ex Director de IESLAC-UNESCO, en Congreso Bianual de la OUI (Organización Universitaria Interamericana) Veracruz, México en el año 2003. 


\title{
Ronny Correa Q.
}

\section{Bibliografía}

\author{
ALBORNOZ, Orlando \\ 1993 Education and Society in Latin America, Londres, Macmillan. \\ AROSEMENA, Guillermo \\ 1998 Hacia un nuevo Ecuador, Primera Edición, Guayaquil-Ecuador. \\ BRUNNER, José Joaquín \\ 1990 Evaluación y financiamiento de la educación superior en América Latina: Bases \\ para un nuevo contrato, Santiago de Chile, FLACSO.
}

1989 Recursos humanos para la investigación en América Latina Santiago de Chile, FLACSO.

2003 Aseguramiento de la calidad y nuevas demandas sobre la educación superior en América Latina", en CNA, Educación superior, calidad y acreditación, Tomo I, Consejo Nacional de Acreditación, Bogotá.

CORREA, Ronny

2006 "Aplicación de un modelo de innovación y estrategias de desarrollo científico de la Universidad Ecuatoriana: "La experiencia de los CITTES de la Universidad Técnica Particular de Loja (UTPL)", Congreso Internacional de Innovación Universitaria PUCE-Quito.

GRILO, E. M.

1990 Las relaciones Universidad - Sector Productivo nos países da América Latina, Revista Iberoamericana de Enseñanza de Ingeniería, vol. 3, N. 1.

JONES, Glenn

2000 Ciberescuelas: El renacimiento de la educación, Caber Publishing Group, Inc., Colorado-EE.UU.

LÓPEZ, Francisco

2006 América Latina y el Caribe: Globalización y Educación Superior, Barcelona.

RAMA, G.,

$1978 \quad$ Notas acerca de la expansión universitaria, el mercado de empleo y las prácticas académicas, Proyecto Desarrollo y Educación en América Latina, Santiago: UNESCO/CEPAL/PNUD.

ROMERO, L. M. \& MUÑOZ, J.

1997 Investigación, ética y Universidad en Latinoamérica, II Encuentro de la Red de Universidades Andinas, Cuzco, 1997.

ROMERO, Luis Miguel

2001 El papel de los centros de transferencia de tecnología y de la educación a distancia, jornada "Reforma universitaria en debate", Asunción-Paraguay.

2006 Seminario Internacional de Evaluación y Acreditación de Instituciones de las Educación Superior a Distancia, Santiago, República Dominicana. 
Universidad e investigación en el contexto del mercado del conocimiento

RUBIO, María José

2002 Centro virtual para el desarrollo de estándares de calidad para la educación superior a distancia en América Latina y el Caribe (Memoria), Universidad Técnica Particular de Loja, Ecuador.

SILVIO, José

2000 La Virtualización de la Universidad ¿cómo podemos transformar la educación superior con la tecnología?, IESALC-UNESCO.

TÜNNERMANN, Carlos

La educación superior en América Latina y el Caribe en su contexto económico, político y social.

UNESCO

2002 Educación Hoy N. 3, Boletín Sector Educación.

2003 Educación Hoy N. 5, Boletín Sector Educación.

UTPL

Documentos varios.

VESSURI, M. C.

1993 Desafíos de la educación superior en relación con la formación y la investigación ante los procesos económicos actuales y los nuevos desarrollos tecnológicos, Publicado en la Revista Iberoamericana de Educación $\quad$ N. 2: Educación, Trabajo y

DIARIO EL COMERCIO Empleo. Mayo-Agosto 1993.

199901 de Febrero.

DIARIO HOY

200531 de Agosto.

\section{Sitios web consultados}

- http:/www.conesup.net

- http://www.cyted.org

- http://www.ricyt.org

- http://www.fundacyt.org

- http://www.utpl.edu.ec 